\title{
环境因子对浮游甲壳动物群落功能多样性的影响“
}

\author{
王松波 ${ }^{* * *}$, 史昭锦 ${ }^{1}$, 耿 红 $^{2}$, 吴来燕 ${ }^{1}$, 曹艳敏 ${ }^{1}$ \\ (1: 中南民族大学资源与环境学院, 武汉 430074) \\ (2: 中南民族大学生命科学学院, 武汉 430074)
}

摘 要: 基于功能性状的生物多样性分析是当前生态学研究中的常用方法之一. 浮游动物作为水生态系统食物网的重要 组成成分, 其功能多样性受关注的程度远不如其重要性. 本文通过对 14 个水体浮游甲壳动物群落结构的研究, 分析了环 境因子对其功能多样性指数, 包括功能丰富度 (FRic)、功能均匀度 (FEve)、功能离散度 (FDiv) 和功能分散度 (FDis), 以 及功能群分布的影响. 结果显示, 亲缘关系近的物种 (在分类学上相近) 由于具有相似的功能性状, 均很好地聚为一类. 相 关分析显示, FRic F E v e 和 FDiv 与环境因子之间不存在显著的相关关系; FDis 与总溶解性氮、总溶解性磷、氨氮和悬浮物 $\mathrm{C} / \mathrm{P}$ 比存在显著的相关关系. 回归分析发现, 随着水体总溶解性磷浓度的上升, 浮游甲壳动物的物种数表现出下降趋势, 功能群数量、功能群 Shannon-Wiener 指数和 FDis 均显著下降; 另外, FRic 和 FDis 与功能群数量均呈显著正相关. 这些结 果表明水体富营养化能促进浮游甲壳动物群落功能性状的趋同, 环境选择是群落结构变异的主要驱动力之一.

关键词: 功能性状;功能群; 聚类分析; 环境滤过; 浮游甲壳动物

\section{Effects of environmental factors on the functional diversity of crustacean zooplankton community*}

\author{
Wang Songbo $^{1 * *}$, Shi Zhaojin ${ }^{1}$, Geng Hong ${ }^{2}$, Wu Laiyan ${ }^{1}$ \& Cao Yanmin ${ }^{1}$ \\ (1: College of Resources and Environmental Science, South-Central University for Nationalities, Wuhan 430074, P.R.China) \\ (2: College of Life Sciences, South-Central University for Nationalities, Wuhan 430074, P.R.China)
}

\begin{abstract}
Methods based on functional traits are widely used in current biodiversity studies. As an important component of aquatic food web, the attention paid on zooplankton by researchers is disproportional to its functional importance. In the present study, the effects of environmental factors on functional diversity indices as well as on distribution of functional groups of crustacean zooplankton communities in 14 waterbodies were investigated. The results showed that species with similar functional traits were grouped together and phylogenetically related. Correlation analysis found no significant correlation between functional richness (FRic), functional evenness (FEve), functional divergence (FDiv) and environmental factors. However, significant relationships were found for functional dispersion (FDis) in relation to total dissolved nitrogen, total dissolved phosphorus( TDP), ammonia nitrogen and seston $\mathrm{C} / \mathrm{P}$ ratio. Regression analysis found that except species richness, number and Shannon-Wiener index of functional groups as well as FDis all declined significantly with the increasing concentration of TDP. In addition, FRic and FDis were both positively correlated with the number of functional groups. These results suggest that eutrophication induces the clustering of functional traits of crustacean zooplankton, and environmental filtering is one of the major forces in driving the formation of crustacean zooplankton community structure.
\end{abstract}

Keywords: Functional trait; functional group; cluster analysis; environmental filtering; crustacean zooplankton

物种多样性是生态系统生产力、稳定性、可人侵性和养分动态变化的主要决定因子 ${ }^{[1]}$. 传统的物种多样 性测定一般以物种数目和丰度为基础 (如物种丰富度、Shannon-Wiener 指数), 近 20 年来以功能性状和系统

* 2020-09-27 收稿; 2020-10-26 收修改稿.

国家自然科学基金项目 (31200361) 和中南民族大学中央高校专项基金项目 (CZY16012，17016)联合资助.

** 通信作者; E-mail: wangsb18@163.com. 
发育为特征的多样性分析逐渐成为生态学研究中的常用手段. 功能多样性 (functional diversity) 是对一个群 落中物种功能性状变化范围和分布的度量. 在此概念出现之前, 群落生态学研究者通常忽略不同物种在生 理、生态和形态特征等方面存在的极大差异, 用物种丰富度代表生物多样性, 这在生物多样性与生态系统功 能关系的研究中尤为常见. 近年来的许多研究发现, 生态系统功能不仅依赖于物种数目, 而且依赖物种所具 有的功能性状, 功能多样性才是与生态过程密切相关的生物多样性因素 ${ }^{[2-3]}$, 在预测生态系统功能时甚至能 超越物种丰富度 ${ }^{[4-5]}$.

功能性状是物种对外界环境长期响应与适应后所呈现出来的特征, 不同的功能性状体现了物种不同的 生态适应性. 经过多年发展, 不同生物类群的功能性状被广泛应用于各种研究. 最常见的植物功能性状包括 叶片形态特征 (如叶片厚度、比叶面积、叶片 $\mathrm{C} / \mathrm{N}$ 、气孔密度、光合速率等)、根属性 (根大小、根长度、比根长 等) 及种子大小和质量 ${ }^{[6-7]}$; 鱼类功能性状有头长、眼睛大小、上/下领长度、鰓耙数目和长度等 ${ }^{[8]}$. 相对于上 述生物所具有的可量化指标, 小型生物所报道的定量指标偏少, 如藻类常用的功能性状中, 体积大小、最大 长度为分类变量, 吞噬性、运动性和对硅的需求为名义变量 ${ }^{[9]}$; 浮游甲壳动物常用的功能性状仅体长为定量 指标,其余 (如摄食类型、栖息生境、食性)均为分类指标 ${ }^{[10]}$. 现代分子生物学证实了物种的功能性状具有相 当的进化保守性, 亲缘关系越近的物种,其功能性状也越相似 ${ }^{[11-12]}$.

与物种多样性指数类似, 功能多样性也可以从不同的方面来描述, 常见的有功能丰富度 (functional richness, FRic) 、功能均匀度 (functional evenness, FEve)、功能离散度 (functional divergence, FDiv) 和功能分散度 (functional dispersion, FDis) 等. FRic 表示群落中物种占据的功能空间的大小, FEve 表示功能性状在功能空 间分布的均匀程度, FDiv 表示群落丰度在功能空间的分布对功能性状离散程度的影响 ${ }^{[13]} ; F D i s$ 表示功能空 间中的各个物种到质心 ( centroid) 的加权平均距离 ${ }^{[14]}$. 这些指数被广泛运用于各种生态学问题的研究, 如群 落构建机制、生物多样性与生态系统功能关系、环境因子对生物群落结构的影响等. 基于生态位理论的群落 构建学说认为, 环境滤过 (environmental filtering) 将导致群落中物种的功能性状趋同 (clustering), 亦即功能 多样性降低. 例如, 湖泊中浮游甲壳动物的功能丰富度和底栖动物的功能分散度均随水体营养水平的上升 而下降 ${ }^{[15-16]}$. 在干旱地区, 土壤水分和含盐量促进了植被叶片面积和氮含量的趋同, 群落功能离散度与水 分呈显著负相关 ${ }^{[17]}$. Bassler 等 ${ }^{[18]}$ 研究发现, 苔藓群落的功能分散度随海拔的升高而降低.

目前有关功能多样性在生态学中的应用习见于陆地生态系统. 浮游动物作为水生态系统中重要的初级 消费者, 其功能多样性受学者关注的程度要远低于鱼类、底栖动物和藻类, 直到 2007 年才有第一篇关于浮 游甲壳动物功能性状的系统描述 ${ }^{[19]}$, 这可能一方面与其个体小、功能性状难以定量量化, 另一方面与其物种 数目少, 存在明显的季节变化和空间分布特征 (如沿岸带和敞水区) 有关. 虽然国际上的相关研究陆续在发 表, 但国内对其的研究仅限于少量的几篇文献 ${ }^{[11,20]}$. 本文通过对 14 个水体浮游甲壳动物群落结构的研究, 分析了环境因子对其功能多样性指数 (包括 FRic、FEve、FDiv 和 FDis) 和功能群分布的影响, 目的在于抛砖 引玉, 让更多的研究者运用功能性状来探讨浮游动物群落多样性的维持、变化机制.

\section{1 研究方法}

\section{1 研究地点}

本研究选取湖北省范围内 14 个水体进行样 品采集,其中湖泊 4 个, 水库 10 个. 野外采样分 别在 2014 年和 2015 年的 4 月 20 日-5 月 9 日进 行. 具体的环境因子采样和分析过程已在王松波 等研究中 ${ }^{[21]}$ 进行了详细的描述, 本文对其进行了 归纳总结并不再赘述 (表 1).

\section{2 浮游甲壳动物采样及计数}

浮游甲壳动物样品通过 $64 \mu \mathrm{m}$ 浮游生物网 的垂直拖曳来采集, 上提速度约为 $0.5 \mathrm{~m} / \mathrm{s}$, 采样 深度为沉积物界面之上 $0.5 \mathrm{~m}$ 至水面. 采集的样
表 1 采样水体的环境因子“

Tab.1 Environmental factors in the sampled water bodies

\begin{tabular}{lccccc}
\hline 环境因子 & \multicolumn{5}{c}{ 平均值标准差最小值最大值 变异系数 } \\
\hline $\mathrm{TDN} /(\mathrm{mg} / \mathrm{L})$ & 1.44 & 0.69 & 0.55 & 3.24 & 0.48 \\
$\mathrm{TDP} /(\mathrm{mg} / \mathrm{L})$ & 0.072 & 0.032 & 0.020 & 0.130 & 0.44 \\
$\mathrm{Chl} . a /(\mu \mathrm{g} / \mathrm{L})$ & 13.8 & 14.0 & 1.1 & 52.8 & 1.02 \\
$K_{\mathrm{d}} / \mathrm{m}^{-1}$ & 1.13 & 0.73 & 0.34 & 3.33 & 0.65 \\
$\mathrm{NH}_{3}-\mathrm{N} /(\mathrm{mg} / \mathrm{L})$ & 0.67 & 0.33 & 0.16 & 1.06 & 0.50 \\
$\mathrm{C} / \mathrm{N}$ 比 & 8.1 & 1.9 & 6.0 & 12.2 & 0.23 \\
$\mathrm{C} / \mathrm{P}$ 比 & 139 & 58 & 59 & 307 & 0.41 \\
\hline
\end{tabular}

$* \mathrm{TDN}$ : 总溶解性氮; TDP : 总溶解性磷; Chl. $a$ : 叶绿素 $a ; K_{\mathrm{d}}$ : 水下光照漫射衰减系数; $\mathrm{C} / \mathrm{N}$ 和 $\mathrm{C} / \mathrm{P}$ 比分别为经 $64 \mu \mathrm{m}$ 滤网 过滤的悬浮物 $\mathrm{C} / \mathrm{N}$ 比和 $\mathrm{C} / \mathrm{P}$ 比. 
品用甲醛保存, 最终浓度为 $5 \%$. 物种组成和密度通过显微镜进行鉴定和计数, 并记录每一物种的最大体长. 枝角类和桡足类的鉴定参考工具书 ${ }^{[22-23]}$. 每个水体采集 3 个样品, 取其密度平均值用于分析.

\section{3 浮游甲壳动物的功能性状}

浮游甲壳动物的功能性状反映了其在水环境中的生态适应性. 参考 Vogt 等 ${ }^{[10]}$ 的研究, 本文选取 5 个功 能性状用于分析,包括:体长 (body length)、摄食类型 (feeding type)、捕食者防御 (predator defense)、栖息地 ( habitat type) 和食性 (trophic group). 在这 5 个指标中, 仅体长为连续型变量, 其余 4 个均为分类变量. 由于 浮游甲壳动物的个体较小, 有关其生理、摄食率、种群增长率等的数据难于从文献中获取, 而体长的大小又 与这些过程直接相关, 故可用体长来间接反映这些重要的生理生态过程. 摄食类型反映了物种获取食物的 方式, 分为 6 种:Bosmina 型 (以水平游泳和不太发达的过滤胸肢为主要特征)、Chydorus 型 (以刮食物体表面 的藻类颗粒为主要特征)、Daphnia 型 (在固定的地方通过第三和第四对胸肢过滤摄食)、Sida 型 (与 Daphnia 型类似, 不同的是利用前 5 对胸肢过滤)、捕食型 (raptorial, 主动捕获猎物)、悬停型 (stationary suspension, 在 一个相对固定的地方悬停摄食). 捕食者防御反映物种面对捕食者时的行为响应, 包括 4 种类型, 无响应、快 速游泳、减慢游泳、中止游泳并跳跃逃离. 栖息地反映物种生活的生境, 分为 2 种, 沿岸带型 (包括底栖型) 和 敞水区型. 食性分为 3 种, 植食性、杂食性和肉食性. 5 个指标除了体长是直接测定的外, 其余指标均通过查 找工具书 ${ }^{[22-23]}$ 获得, 本研究中各物种的具体功能性状见表 2 .

表 2 浮游甲壳动物的功能性状

Tab.2 Functional traits of crustacean zooplankton

\begin{tabular}{|c|c|c|c|c|c|}
\hline 物种 & 体长/mm & 摄食类型 & 捕食者防御 & 栖息地 & 食性 \\
\hline 透明薄皮溞 Leptodora kindti & 2.55 & 捕食型 & 无响应 & 敞水区 & 肉食性 \\
\hline 晶莹仙达溞 Sida crystallina & 0.90 & Sida 型 & 无响应 & 沿岸带 & 植食性 \\
\hline 盔形溞 Daphnia galeata & 1.00 & Daphnia 型 & 快速游泳 & 敞水区 & 植食性 \\
\hline 微型裸腹溞 Moina micrura & 0.68 & Daphnia 型 & 快速游泳 & 敞水区 & 植食性 \\
\hline 角突网纹溞 Ceriodaphnia cornuta & 0.45 & Daphnia 型 & 快速游泳 & 敞水区 & 植食性 \\
\hline 方形网纹溞 Ceriodaphnia quadrangula & 0.50 & Daphnia 型 & 快速游泳 & 敞水区 & 植食性 \\
\hline 老年低额溞 Simocephalus vetulus & 0.75 & Daphnia 型 & 快速游泳 & 沿岸带 & 植食性 \\
\hline 尖额溞 Alona sp. & 0.40 & Chydorus 型 & 无响应 & 沿岸带 & 植食性 \\
\hline 盘肠溞 Chydorus sp. & 0.30 & Chydorus 型 & 无响应 & 沿岸带 & 植食性 \\
\hline 钩足平直溞 Pleuroxus hamulatus & 0.48 & Chydorus 型 & 无响应 & 沿岸带 & 植食性 \\
\hline 简弧象鼻溞 Bosmina coregoni & 0.33 & Bosmina 型 & 减慢游泳 & 敞水区 & 植食性 \\
\hline 颈沟基合溞 Bosminopsis deitersi & 0.38 & Bosmina 型 & 减慢游泳 & 沿岸带 & 植食性 \\
\hline 秀体溞 Diaphanosoma sp. & 0.68 & Sida 型 & 中止游泳并跳跃 & 敞水区 & 植食性 \\
\hline 台湾温剑水蚤 Thermocyclops taihokuensis & 0.80 & 捕食型 & 中止游泳并跳跃 & 敞水区 & 肉食性 \\
\hline 中剑水蚤 Mesocyclops sp. & 0.80 & 捕食型 & 中止游泳并跳跃 & 敞水区 & 肉食性 \\
\hline 锯缘真剑水蚤 Eucyclops serrulatus & 0.85 & 捕食型 & 中止游泳并跳跃 & 沿岸带 & 植食性 \\
\hline 近邻剑水蚤 Cyclops vicinus & 1.13 & 捕食型 & 中止游泳并跳跃 & 敞水区 & 杂食性 \\
\hline 汤匙华哲水蚤 Sinocalanus dorrii & 1.58 & 悬停型 & 中止游泳并跳跃 & 敞水区 & 杂食性 \\
\hline 特异荡镖水蚤 Neutrodiaptomus incongruens & 1.05 & 悬停型 & 中止游泳并跳跃 & 敞水区 & 杂食性 \\
\hline 右突新镖水蚤 Neodiaptomus schmackeria & 1.58 & 悬停型 & 中止游泳并跳跃 & 敞水区 & 杂食性 \\
\hline 球状许水蚤 Schmackeria forbesi & 1.08 & 悬停型 & 中止游泳并跳跃 & 敞水区 & 杂食性 \\
\hline 长江新镖水蚤 Neodiaptomus yangtsekiangensis & 1.35 & 悬停型 & 中止游泳并跳跃 & 敞水区 & 杂食性 \\
\hline
\end{tabular}

\section{4 功能多样性指数}

功能丰富度 (FRic) 指数以凸包体积 (convex hull volume) 来衡量, 表示在多维功能空间中能将所有物种 囊括在内的最小多面体体积,在二维空间中则表现为多边形面积. 该凸包体的计算基于 “Quickhull” 算法, 是 一个复杂的过程, 必须依赖计算机完成 ${ }^{[24]}$.

功能均匀度 $(F E v e)$ 指数 ${ }^{[24]}$ : 


$$
\begin{aligned}
& F E v e=\frac{\sum_{l=1}^{S-1} \min \left(P E W_{l}, \frac{1}{S-1}\right)-\frac{1}{S-1}}{1-\frac{1}{S-1}} \\
& P E W_{l}=\frac{E W_{l}}{\sum_{l=1}^{S-1} E W_{l}} \\
& E W_{l}=\frac{\operatorname{dist}(i, j)}{w_{i}+w_{j}}
\end{aligned}
$$

式中, $w_{i} 、 w_{j}$ 表示物种 $i v j$ 的相对丰度, $\operatorname{dist}(i, j)$ 表示在最小生成树 (minimum spanning tree, MST) 中两相邻物 种 $i$ 和 $j$ 之间的欧氏距离, $E W_{l}$ 表示经相对丰度加权处理后的均匀度; $l$ 表示在 MST 中连接两相邻物种的树枝 数量; $P E W_{l}$ 表示局部加权均匀度; $S$ 表示物种丰富度.

功能离散度 $(F D i v)$ 指数 ${ }^{[24]}$ :

$$
\begin{gathered}
\text { FDiv }=\frac{\Delta d+\overline{d G}}{\Delta|d|+\overline{d G}} \\
\Delta|d|=\sum_{i=1}^{S} w_{i}\left|d G_{i}-\overline{d G}\right| \\
\Delta d=\sum_{i=1}^{S} w_{i}\left(d G_{i}-\overline{d G}\right) \\
\overline{d G}=\frac{1}{S} \sum_{i=1}^{S} d G_{i} \\
d G_{i}=\sqrt{\sum_{k=1}^{T}\left(x_{i k}-g_{k}\right)^{2}} \\
g_{k}=\frac{1}{V} \sum_{i=1}^{V} x_{i k}
\end{gathered}
$$

功能分散度 $(F D i s)$ 指数 ${ }^{[14]}$ :

$$
\begin{gathered}
\text { FDis }=\sum_{i=1}^{S} w_{i} \cdot z_{i} \\
c=\left[c_{k}\right]=\sum_{i=1}^{S} w_{i} \cdot x_{i k}
\end{gathered}
$$

式中, $x_{i k}$ 表示物种 $i$ 的第 $k$ 个性状值; $V$ 表示形成凸包体的顶点个数; $g_{k}$ 表示性状 $k$ 的重心 $T$ 表示性状数量; $S$ 表示物种丰富度; $d G_{i}$ 表示物种 $i$ 到凸包体重心 (the center of gravity) 的距离; $\overline{d G}$ 表示物种距离重心的平均 距离; $w_{i}$ 表示物种 $i$ 的相对丰度; $\Delta d$ 表示经丰度加权处理后的物种 $i$ 相对于平均距离的离散度; $\Delta|d|$ 表示经 丰度加权处理后的物种 $i$ 相对于平均距离的绝对离散度; $c_{k}$ 表示群落全部物种第 $k$ 个性状值的质心 $; z_{i}$ 表示 物种 $i$ 到质心的距离.

\section{5 统计分析}

物种对之间的相异矩阵采用 Gower 距离构建. 聚类分析采用非加权组平均法 (UPGMA), 通过 “hclust” 函数实现. 功能多样性指数通过 FD 软件包中的 “ dbFD” 函数实现, 上述分析均依赖 R 3.6.1. 相关分析和线 性回归分析通过 SPSS 17.0 软件完成.

\section{2 结果与分析}

\section{1 浮游甲壳动物功能群}

基于 Gower 距离和非加权组平均法的聚类分析见图 1. 结果显示: 亲缘关系近的物种 (在分类学上相近) 由于具有相似的功能性状,均很好地聚为一类, 如颈沟基合溞和简弧象鼻掻, 角突网纹掻和方形网纹溞. 另 外, 在分类学上隶属于某一科或目的物种也能很好地聚集在一起, 如溞科的角突网纹溞、方形网纹溞、盔形 


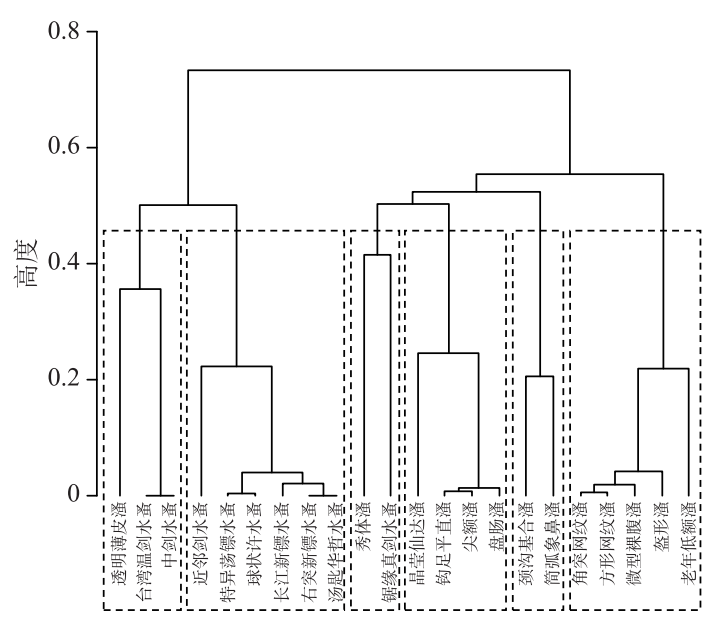

图 1 基于浮游甲壳动物功能性状的聚类分析

Fig.1 Cluster analysis based on functional traits of crustacean zooplankton
溞、老年低额溞, 哲水蚤目中的汤匙华哲水蚤、特异 荡镖水蚤、右突新镖水蚤、球状许水蚤和长江新镖 水蚤. 此外, 生活在沿岸带, 与水草关系紧密的晶芗 仙达溞、钩足平直溞、尖额溞和盘肠溞聚在一起; 肉 食性种类, 如透明薄皮溞、中剑水蚤和台湾温剑水 虫聚为一类. 为方便研究, 根据优势种组成, 我们将 上述 22 个种属分为 6 个功能群, 分别称之为 Mesocyclops 群组、Sinocalanus 群组、Diaphanosoma 群组、 Chydorus 群组、Bosmina 群组和 Daphnia 群组 (图 1). 在这些采样水体中, 浮游甲壳动物的功能群数 量变化范围为 $2 \sim 6$, 均值为 4 . 除火连畈水库 Mesocyclops 群组和 Diaphanosoma 群组占优势外, Daphnia 群组和 Sinocalanus 群组在其余水体中合计 占到了总密度的 $45 \% \sim 96 \%$ ( 图 2).

\section{2 浮游甲壳动物功能多样性指数}

在 16 个采样水体中, FRic 变化范围为 0.085 0.673 ,均值 0.374 , 最大值出现在 2015 年的吴岭水 库, 最小值出现在金沙水库. FEve 均值为 0.489 , 最

大值和最小值分别出现在惠亭水库 $(0.722)$ 和罗桥水库 (0.192). FDiv 变化范围为 $0.670 \sim 0.982$, 均值 0.868 , 最大值出现在 2015 年的吴岭水库,最小值出现在牛山湖. FDis 均值为 0.227 ,最大值出现在 2014 年的吴岭 水库 (0.350), 最小值出现在北郊水库 (0.107). 火连畈水库由于物种数小于经主坐标分析后选取的特征轴 数,无法进行 FRic、FEve 和 FDiv 的计算 (表 3).

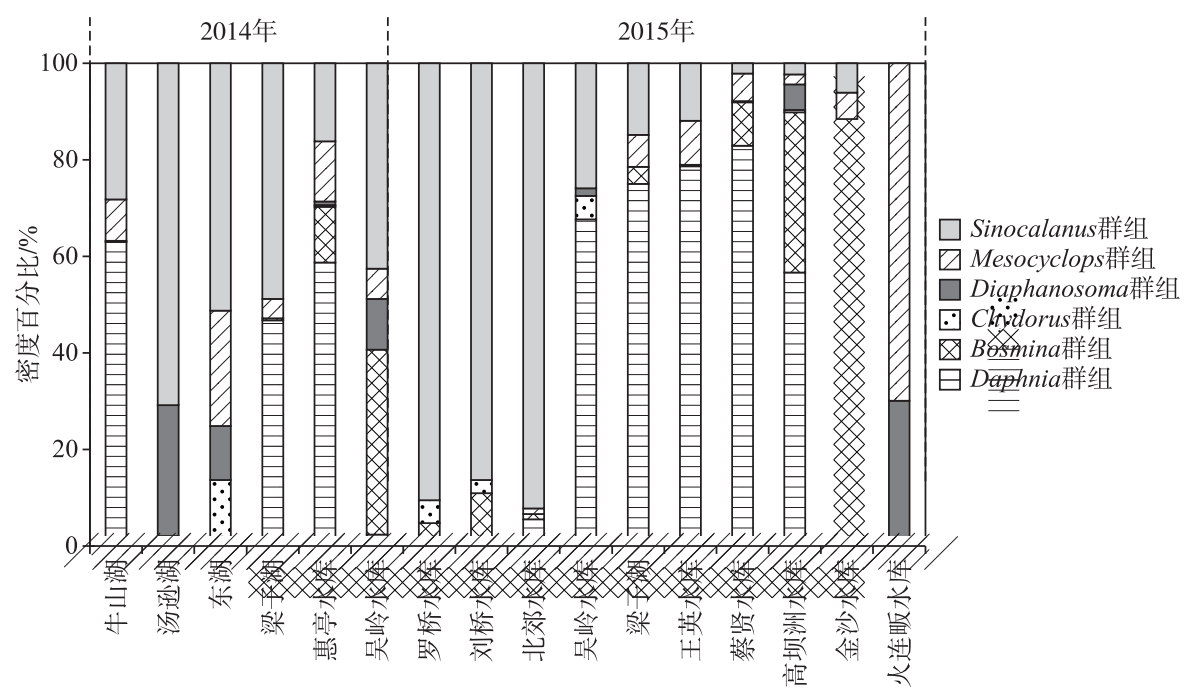

图 2 各采样水体功能群密度百分比组成

Fig.2 Percentage compositions of functional groups in the sampled water bodies

相关分析显示, FRic、FEve 和 FDiv 与环境因子之间不存在显著的相关关系 $(P>0.05)$. FDis 与 TDN、 $\mathrm{TDP} 、 \mathrm{NH}_{3}-\mathrm{N}$ 和 $\mathrm{C} / \mathrm{P}$ 比均存在显著的相关关系 $(P<0.05)$ (表 4). 线性回归分析发现, 随着水体 TDP 浓度的 增加, 浮游甲壳动物的物种数表现出下降趋势 ( $\left.R^{2}=0.21, P=0.073\right)$, 功能群数量 $\left(R^{2}=0.36, P=0.014\right)$ 、功 能群 Shannon-Wiener 指数 $\left(R^{2}=0.60, P<0.001\right)$ 和 $F D i s\left(R^{2}=0.66, P<0.001\right)$ 均显著下降 (图 3). 另外, FRic 
和 FDis 均随着功能群数量的增加而显著变大 $(P<0.05)$ ( 图 4).

表 3 浮游甲壳动物的功能多样性指数

Tab.3 Functional diversity indices of crustacean zooplankton

\begin{tabular}{llllll}
\hline 采样时间 & 地点 & FRic & FEve & FDiv & FDis \\
\hline \multirow{2}{*}{2014 年 } & 牛山湖 & 0.479 & 0.469 & 0.670 & 0.303 \\
& 汤逊湖 & 0.188 & 0.684 & 0.799 & 0.189 \\
& 东湖 & 0.619 & 0.393 & 0.781 & 0.306 \\
& 梁子湖 & 0.311 & 0.432 & 0.981 & 0.330 \\
& 惠亭水库 & 0.512 & 0.722 & 0.883 & 0.290 \\
& 吴岭水库 & 0.217 & 0.544 & 0.968 & 0.129 \\
& 罗桥水库 & 0.256 & 0.192 & 0.883 & 0.206 \\
& 刘桥水库 & 0.288 & 0.556 & 0.787 & 0.107 \\
& 北郊水库 & 0.212 & 0.230 & 0.982 & 0.280 \\
& 吴岭水库 & 0.673 & 0.511 & 0.967 & 0.230 \\
& 梁子湖 & 0.311 & 0.499 & 0.955 & 0.210 \\
& 王英水库 & 0.311 & 0.430 & 0.941 & 0.141 \\
& 蔡贤水库 & 0.509 & 0.694 & 0.683 & 0.258 \\
& 高坝洲水库 & 0.633 & 0.407 & 0.957 & 0.122 \\
& 金沙水库 & 0.085 & 0.572 & - & 0.173 \\
\hline
\end{tabular}

一表示火连畈水库由于物种数小于经主坐标分析后选取的特征轴数, 无法进行 FRic、FEve 和 FDiv 的计算.
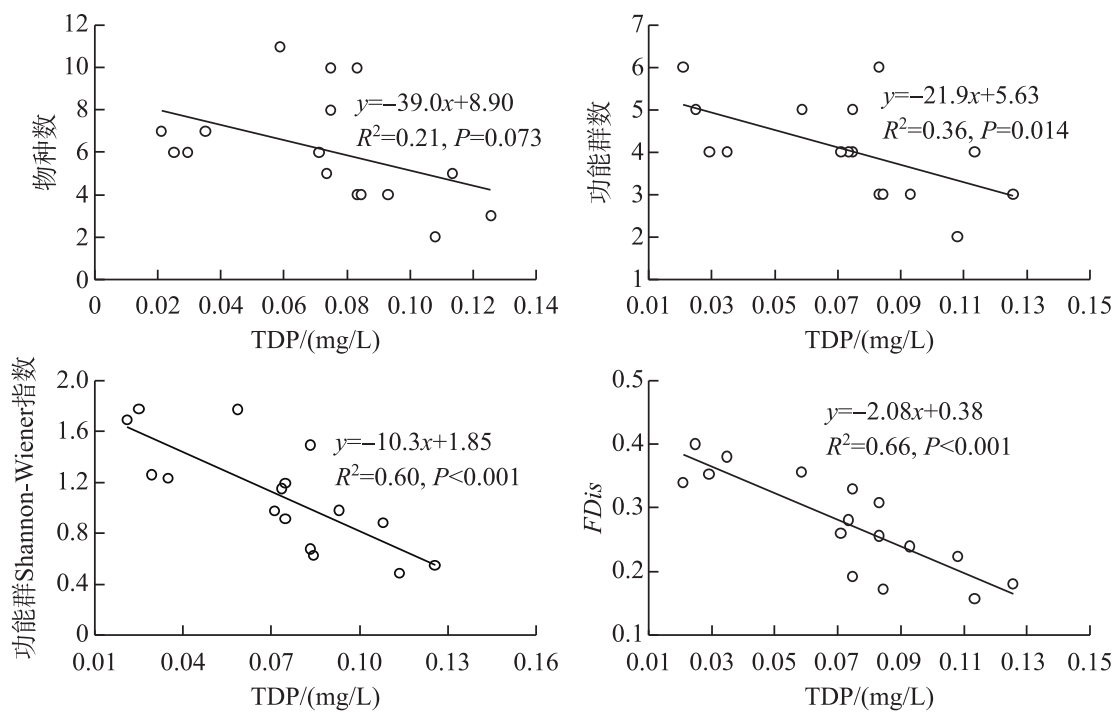

图 3 物种数、功能群数、功能群 Shannon-Wiener 指数和功能分散度 (FDis) 与 TDP 浓度之间的回归分析

Fig.3 Regression analyses of species number, number and Shannon-Wiener index of functional groups as well as FDis to TDP concentration

\section{3 讨论}

功能多样性指数从不同方面刻画了群落功能性状的分布模式. FRic 表示一个群落中的物种所占据的功 能空间的大小, 该值一般随物种丰富度的增多而变大, 其余 3 个指数与物种丰富度无关 ${ }^{[1,24-25]}$. 在本研究中, 
表 4 功能多样性指数与环境因子之间的 Spearman 相关分析

Tab.4 Spearman correlation between functional diversity indices and environmental factors

\begin{tabular}{lllcc}
\hline 环境因子 & FRic & FEve & FDiv & FDis \\
\hline TDN & n.s. & n.s. & n.s. & $-0.609^{*}$ \\
TDP & n.s. & n.s. & n.s. & $-0.832^{* *}$ \\
Chl. $a$ & n.s. & n.s. & n.s. & n.s. \\
$K_{\mathrm{d}}$ & n.s. & n.s. & n.s. & n.s. \\
$\mathrm{NH}_{3}-\mathrm{N}$ & n.s. & n.s. & n.s. & $-0.645^{* *}$ \\
$\mathrm{C} / \mathrm{N}$ & n.s. & n.s. & n.s. & n.s. \\
$\mathrm{C} / \mathrm{P}$ & n.s. & n.s. & n.s. & $0.570^{*}$ \\
\hline
\end{tabular}

* 表示显著相关, $P<0.05 ; * *$ 表示极显著相关, $P<0.01 ;$ n.s.表示相关性不显著, $P>0.05$.
FRic 与物种丰富度呈显著正相关 $(R=0.77, P=0.001)$, 但随着水体 TDP 浓度的增大, 物种丰富度呈不显著的下 降趋势, FRic 与 TDP 浓度之间也呈不显著的负相关关系 $(R=-0.31, P=0.259)$. 已有的研究发现, 浮游甲壳动物 的功能丰富度与 TP 浓度之间呈单峰分布或线性负相关 的模式 ${ }^{[15,26]}$. 这种分布模式实际上与物种丰富度随水体 营养状态或初级生产力的分布具有一致性, 如 Jeppesen 等 ${ }^{[27]}$ 发现浮游动物的物种数随水体营养水平的上升而 下降; Dodson 等 ${ }^{[28]}$ 发现浮游动物的物种数与初级生产力 之间的关系为单峰模型. 虽然 FRic 的大小受物种丰富度 的直接影响, 但多的物种数量并不总是代表高的 FRic, 因为可能有圥余物种存在. 例如 Bässler 等 ${ }^{[18]}$ 研究发现 随着海拔的增加, 苔藓群落的功能分散度下降, 但物种 丰富度反而上升,主要表现为喜好高湿环境和行无性繁 殖模式种类数的增加. 在一个水体中如果同时出现汤匙

华哲水蚤、右突新镖水蚤和长江新镖水蚤, 与只有其中的一个物种相比, FRic 可能并不会受到多大影响. 在 本研究中, 功能群数量随着 TDP 浓度的增加而下降, 而且 FRic 与功能群数量之间呈显著正相关. 在水体富 营养化的过程中, 浮游甲壳动物功能群数量的下降说明环境选择促进了它们功能性状的趋同, 同时也表明 它们对资源充分利用能力的下降, 这可能也是富营养水体中浮游动植物间能量传递效率下降的原因之一.
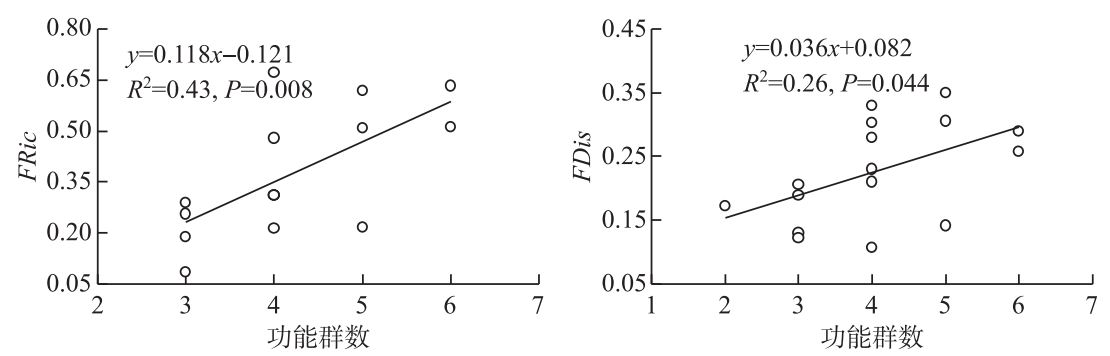

图 4 浮游甲壳动物功能丰富度 (FRic) 和功能分散度 (FDis) 与功能群数量之间的回归分析

Fig.4 Regression analyses of FRic and FDis to the number of functional groups of crustacean zooplankton

融合物种丰富度与丰度的 FEve 和 FDiv 也不受所选环境因子的影响. 一般来说, 如果优势种分布在功能 空间的边缘,FDiv 就大, 表明生态位分化明显, 物种间资源竞争较弱; 反之, 如果优势种靠近功能空间重心, $F D i v$ 就小,物种间资源竞争激烈 ${ }^{[1,24]}$. 在本研究中, FDiv 普遍偏大, 说明群落物种间的竞争较弱. 例如在最 小 FDiv 出现的牛山湖,盔形溞和汤匙华哲水蚤分别占总密度的 $62.5 \%$ 和 $27.9 \%$, 两者虽然都生活在敞水区, 以滤食藻类为主, 但可能通过对食物颗粒大小的选择实现生态位的分化, 前者以小颗粒为主, 后者则以大颗 粒为主 ${ }^{[29]} . F E v e$ 基本分布在 0.5 左右,其大小不仅受物种间距离的影响,还受物种丰度在功能空间分布的影 响. 在上述 FRic 的讨论中, 我们发现水体富营养化导致浮游甲壳动物功能群数量减少, 亦即物种在功能空间 聚集,物种间性状趋同, 对资源的利用能力下降, 理论上FDiv 和 FEve 也会变小,但它们两者的计算还受物种 丰度在功能空间分布的影响, 所以本研究对其大小随营养水平的变化规律很难给出合理的解释. 考虑到这 些水体中依然存在渔业养殖活动, 强烈的下行效应可能导致了这些结果的产生. $\mathrm{Li}^{\text {等 }}{ }^{[30]}$ 认为在长江流域受 人类活动影响强烈的水体中浮游动物的群落结构主要受鱼类捕食的影响, 与营养状态的关系不大. 张晓妆 等 ${ }^{[31]}$ 也认为每年春、秋季渔汛期对经济鱼种进行高强度捕捞, 从而引起其生物量大幅下降, 可能是造成海州 湾鱼类群落优势种和功能均匀度、功能离散度变化的重要原因. 之前的研究发现 ${ }^{[21]}$, 浮游甲壳动物密度和 生物量均与 Chl. $a$ 浓度不存在显著相关性, 且有 $78 \%$ 水体的 TDP 浓度高于 $0.07 \mathrm{mg} / \mathrm{L}$. 在长江中下游的浅水 
湖泊中, 当总磷浓度大于 $0.097 \mathrm{mg} / \mathrm{L}$ 时, 浮游甲壳动物生物量会因鱼类捕食而变得不确定, 由于我们所选择 的水库和湖泊大都存在渔业活动, 鱼类捕食对其群落结构和功能多样性指数的影响不容忽视 ${ }^{[30,32]}$.

FDiv 在计算重心时, 仅用到了构成多面体的顶点 (顶点数 $\leqslant$ 物种数) 对应的物种坐标, 且仅在计算物种 到重心的距离时用到了物种丰度权重 ${ }^{[24]}$. FDis 在计算质心坐标时用的是全部物种坐标, 而且在计算质心坐 标和物种到质心距离时均用到了物种丰度权重 ${ }^{[14]}$, 也就是说 FDis 比 FDiv 更能反映物种在功能空间的聚集 程度. 在本研究中, FDis 不仅与营养盐 ( TDN、TDP、 $\mathrm{NH}_{3}-\mathrm{N}$ ) 浓度呈显著负相关, 而且还与功能群数量呈显著 正相关, 表明富营养化促进了浮游甲壳动物群落功能性状在生态位空间的聚集. Valdivia 等 ${ }^{[33]}$ 在潮间带的 研究也发现, 栖居于严酷环境下 (高潮间带) 的生物群落的 FRic 和 FDis 最低, 表现为性状趋同, 而在温和条 件下 (低潮间带) FRic 和 FDis 最大, 表现为性状发散. 随着水体营养水平的上升, 大型底栖动物群落的功能 性状 $\beta$ 多样性显著下降 ${ }^{[16]}$. 植物与生长和繁殖有关的功能性状的 FDis 随温度和降雨的增加而变大 ${ }^{[34]}$. 这 些结果表明, 群落的 FDis 受群落构建过程 (例如环境滤过) 的直接影响, 水体富营养化带来的物理、化学、生 物等环境的改变最终会影响到浮游动物的群落结构. 另外, FDis 与悬浮物 $\mathrm{C} / \mathrm{P}$ 比呈显著正相关. 在我们研 究的水体中, 悬浮物 $\mathrm{C} / \mathrm{P}$ 比均满足浮游甲壳动物发展对食物质量的要求 ${ }^{[21]}$, 相反过低的悬浮物 $\mathrm{C} / \mathrm{P}$ 比可能 会对浮游甲壳动物的生长造成负面影响 ${ }^{[35]}$, 从而对其群落组成产生选择压力, 所以悬浮物 $\mathrm{C} / \mathrm{P}$ 比的适当增 大可能更有利于多样化物种的共存.

功能群是群落中具有相似功能物种的集合. 在经验性的水生动物功能群划分中, 个体大小、摄食类型和 食性常被用于分组, 如 Krzton 等 ${ }^{[36]}$ 根据这些特征将包括纤毛虫、轮虫和浮游甲壳动物在内的浮游动物群体 分为 6 个功能类群; Heino ${ }^{[37]}$ 通过摄食和行为特点的组合将底栖动物划分为 24 个功能群. 本文依据 5 个功 能性状对浮游甲壳动物的聚类分析发现, 在分类学上相近的物种 (也可粗略地认为在系统发育上亲缘关系 较近 ${ }^{[38]}$, 其功能性状的差异也较小, 更容易形成一个类群, 表明功能性状可能具有进化上的保守性 ${ }^{[11]}$. 以 外形特征作为主要分类依据的枝角类、哲水蚤和剑水蚤本身也隐含着功能性状的应用, 如运动方式和食性. 随着水体 TDP 浓度的上升, 浮游甲壳动物功能群的数量和 Shannon-Wiener 多样性指数均显著下降, 表明沿 着营养盐梯度群落中的物种向更适应当前环境的功能群或优势种集中. Heino ${ }^{[37]}$ 也发现水体中 TP 浓度的增 加降低了底栖动物功能群的多样性和均匀性. 群落构建理论认为环境选择一般会导致群落功能性状的趋同. 在本研究中, 低营养水体以 2 个优势功能群为主, 包括 Daphnia、Sinocalanus、Bosmina 群组的组合, 其密度能 占到总密度的 $75 \%$ 以上; 在中、高营养水平的水体, 如罗桥水库、北郊水库和王英水库, 一个功能群就能占到 总密度的 70\%以上,这些优势功能群基本以 Daphnia 或 Sinocalanus 群组为主 ${ }^{[21]}$. 当 Daphnia 和 Sinocalanus

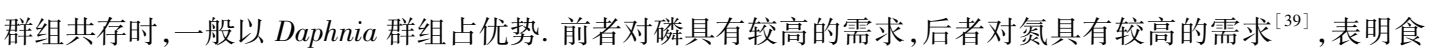
物质量在群落构建中可能占有重要作用.

\section{4 参考文献}

[ 1 ] Tilman D, Isbell F, Cowles JM. Biodiversity and ecosystem functioning. Annual Review of Ecology, Evolution, and Systematics, 2014, 45(1) : 471-493. DOI: 10.1146/annurev-ecolsys-120213-091917.

[ 2 ] Thompson PL, Davies TJ, Gonzalez A. Ecosystem functions across trophic levels are linked to functional and phylogenetic diversity. PLoS One, 2015, 10(2) : e0117595. DOI: 10.1371/journal.pone.0117595.

[ 3 ] Gross N, le Bagousse-Pinguet Y, Liancourt P et al. Functional trait diversity maximizes ecosystem multifunctionality. Nature Ecology \& Evolution, 1(5) : 1-9. DOI: 10.1038/s41559-017-0132.

[ 4 ] Abonyi A, Horváth Z, Ptacnik R. Functional richness outperforms taxonomic richness in predicting ecosystem functioning in natural phytoplankton communities. Freshwater Biology, 2018, 63(2) : 178-186. DOI: 10.1111/fwb.13051.

[ 5 ] Gagic V, Bartomeus I, Jonsson T et al. Functional identity and diversity of animals predict ecosystem functioning better than species-based indices. Proceedings of the Royal Society B: Biological Sciences, 2015, 282(1801) : 20142620. DOI: 10.1098/rspb.2014.2620.

[ 6 ] Liu XJ, Ma KP. Plant functional traits-concepts, applications and future directions. Science in China: Series C, 2015,45 (4) : 325-339. DOI: 10.1360/N052014-00244. [刘晓娟, 马克平. 植物功能性状研究进展. 中国科学: 生命科学, $2015, \mathbf{4 5}(4)$ : 325-339.] 
[ 7 ] He NP, Liu CC, Zhang JH et al. Perspectives and challenges in plant traits: From organs to communities. Acta Ecologica Sinica, 2018, 38(19): 6787-6796. DOI: 10.5846/stxb201710241900. [何念鹏, 刘聪聪, 张佳慧等. 植物性状研究 的机遇与挑战: 从器官到群落. 生态学报, $2018,38(19): 6787-6796$.

[ 8 ] Ingram T, Shurin JB. Trait-based assembly and phylogenetic structure in northeast Pacific rockfish assemblages. Ecology, 2009, 90(9) : 2444-2453. DOI: 10.1890/08-1841.1.

[ 9 ] Weithoff G, Rocha MR, Gaedke U. Comparing seasonal dynamics of functional and taxonomic diversity reveals the driving forces underlying phytoplankton community structure. Freshwater Biology, 2015, 60 (4) : 758-767. DOI: 10.1111/ fwb.12527.

[10] Vogt RJ, Peres-Neto PR, Beisner BE. Using functional traits to investigate the determinants of crustacean zooplankton community structure. Oikos, 2013, 122(12) : 1700-1709. DOI: 10.1111/j.1600-0706.2013.00039.x.

[11] Zhao K. Species diversity of zooplankton in Huaihe River Basin: spatial pattern and formation mechanism [Dissertation]. Shanghai : East China Normal University, 2018. [赵坤. 淮河流域浮游动物分布格局及群落形成机制 [学位论文]. 上 海: 华东师范大学, 2018.]

[12] Kraft NJB, Ackerly DD. Functional trait and phylogenetic tests of community assembly across spatial scales in an Amazonian forest. Ecological Monographs, 2010, 80(3) : 401-422. DOI: 10.1890/09-1672.1.

[13] Mason NWH, Mouillot D, Lee WG et al. Functional richness, functional evenness and functional divergence: The primary components of functional diversity. Oikos, 2005 , 111(1) : 112-118. DOI: 10.1111/j.0030-1299.2005.13886.x.

[14] Laliberté E, Legendre P. A distance-based framework for measuring functional diversity from multiple traits. Ecology, 2010, 91(1) : 299-305. DOI: 10.1890/08-2244.1.

[15] Barnett A, Beisner BE. Zooplankton biodiversity and lake trophic state: Explanations invoking resource abundance and distribution. Ecology, 2007, 88(7) : 1675-1686. DOI: 10.1890/06-1056.1.

[16] Zhang Y, Cheng L, Li KY et al. Nutrient enrichment homogenizes taxonomic and functional diversity of benthic macroinvertebrate assemblages in shallow lakes. Limnology and Oceanography, 2019, 64 (3) : 1047-1058. DOI: 10. 1002/ lno.11096.

[17] Gong YM, Ling HB, Lv G et al. Disentangling the influence of aridity and salinity on community functional and phylogenetic diversity in local dryland vegetation. Science of the Total Environment, 2019, 653: 409-422. DOI: 10.1016/j.scitotenv.2018.10.358.

[18 B Bässler C, Cadotte MW, Beudert B et al. Contrasting patterns of lichen functional diversity and species richness across an elevation gradient. Ecography, 2016, 39(7) : 689-698. DOI: 10.1111/ecog.01789.

[19] Barnett AJ, Finlay K, Beisner BE. Functional diversity of crustacean zooplankton communities: Towards a trait-based classification. Freshwater Biology, 2007, 52(8) : 796-813. DOI: 10.1111/j.1365-2427.2007.01733.x.

[20] Gomes LF, Pereira HR, Gomes ACAM et al. Zooplankton functional-approach studies in continental aquatic environments: A systematic review. Aquatic Ecology, 2019, 53(2) : 191-203. DOI: 10.1007/s10452-019-09682-8.

[21] Wang SB, Geng H, Wu LY et al. Effects of food and physicochemical factors on crustacean zooplankton community structure. J Lake Sci, 2018, 30(3) : 723-731. DOI: 10.18307/2018.0314. [王松波, 耿红, 吴来燕等. 食物和理化因子对 浮游甲壳动物群落结构的影响. 湖泊科学, 2018, 30(3) : 723-731.]

[22] Jiang XZ, Du NS eds. Fauna sinica, crustacea:Freshwater cladocera. Beijing: Science Press, 1979. [蒋笅治, 堵南山. 中国动物志: 淡水枝角类. 北京: 科学出版社, 1979.]

[23] Shen JR ed. Fauna sinica, crustacea: Freshwater copepoda. Beijing: Science Press, 1979. [沈嘉瑞. 中国动物志: 淡水 桡足类. 北京: 科学出版社, 1979.]

[24] Villéger S, Mason NWH, Mouillot D. New multidimensional functional diversity indices for a multifaceted framework in functional ecology. Ecology, 2008, 89(8) : 2290-2301. DOI: 10.1890/07-1206.1.

[25] Mouchet MA, Villéger S, Mason NWH et al. Functional diversity measures: An overview of their redundancy and their ability to discriminate community assembly rules. Functional Ecology, 2010, 24(4) : 867-876. DOI: 10.1111/j.13652435.2010.01695.x.

[26] Nevalainen L, Luoto TP. Relationship between cladoceran (Crustacea) functional diversity and lake trophic gradients. Functional Ecology, 2017, 31(2) : 488-498. DOI: 10.1111/1365-2435.12737.

[27] Jeppesen E, Peder JJ, Søndergaard M et al. Trophic structure, species richness and biodiversity in Danish lakes: Changes 
along a phosphorus gradient. Freshwater Biology, 2000, 45(2) : 201-218. DOI: 10.1046/j.1365-2427.2000.00675.x.

[28] Dodson SI, Arnott SE, Cottingham KL. The relationship in lake communities between primary productivity and species richness. Ecology, 2000, 81(10) : 2662-2679. DOI: 10.1890/0012-9658(2000)081[2662: TRILCB] 2.0.CO;2.

[29] Sommer U, Sommer F, Santer B et al. Complementary impact of copepods and cladocerans on phytoplankton. Ecology Letters, 2001, 4(6) : 545-550. DOI: 10.1046/j.1461-0248.2001.00263.x.

[30] Li Y, Chen FZ. Are zooplankton useful indicators of water quality in subtropical lakes with high human impacts? Ecological Indicators, 2020, 113: 106167. DOI: 10.1016/j.ecolind.2020.106167.

[31] Zhang XZ, Wang J, Xu BD et al. Spatio-temporal variations of functional diversity of fish communities in Haizhou Bay. Chinese Journal of Applied Ecology, 2019, 30(9) : 3233-3244. DOI: 10.13287/j.1001-9332.201909.035. [张晓妆, 王 晶, 徐宾铎等. 海州湾鱼类群落功能多样性的时空变化. 应用生态学报, 2019, 30(9) : 3233-3244.]

[32] Wang SB, Xie P, Wu SK et al. Crustacean zooplankton size structure in aquaculture lakes: Is larger size structure always associated with higher grazing pressure? Hydrobiologia , 2007, 575(1) : 203-209. DOI: 10.1007/s10750-006-0394-7.

[33] Valdivia N, Segovia-Rivera V, Fica E et al. Context-dependent functional dispersion across similar ranges of trait space covered by intertidal rocky shore communities. Ecology and Evolution, 2017, 7 (6) : 1882-1891. DOI: 10. 1002/ ece3.2762.

[34] Schellenberger CD, Gerschlauer F, Pabst H et al. Community-weighted means and functional dispersion of plant functional traits along environmental gradients on Mount Kilimanjaro. Journal of Vegetation Science, 2017, 28(4) : 684-695. DOI: $10.1111 /$ jvs. 12542 .

[35] Elser JJ, Kyle M, Learned J et al. Life on the stoichiometric knife-edge: Effects of high and low food C: P ratio on growth, feeding, and respiration in three Daphnia species. Inland Waters, 2016, 6(2) : 136-146. DOI: 10.5268/IW-6.2.908.

[36] Krztoń W, Kosiba J. Variations in zooplankton functional groups density in freshwater ecosystems exposed to cyanobacterial blooms. Science of the Total Environment, 2020, 730: 139044. DOI: 10.1016/j.scitotenv.2020.139044.

[37] Heino J. Patterns of functional biodiversity and function-environment relationships in lake littoral macroinvertebrates. Limnology and Oceanography, 2008, 53(4) : 1446-1455. DOI: 10.4319/lo.2008.53.4.1446.

[38 ] Heino J, Tolonen KT. Untangling the assembly of littoral macroinvertebrate communities through measures of functional and phylogenetic alpha diversity. Freshwater Biology, 2017, 62(7) : 1168-1179. DOI: 10.1111/fwb.12934.

[39] Moody EK, Wilkinson GM. Functional shifts in lake zooplankton communities with hyper eutrophication. Freshwater Biolo$g y, 2019,64(3):$ 608-616. DOI: 10.1111/fwb.13246. 